
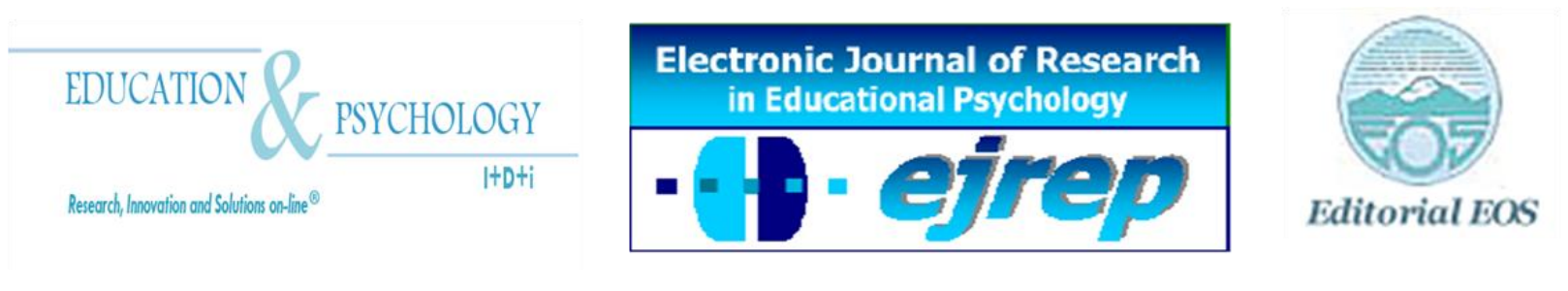

\title{
Aprender a Convivir. Un programa para la mejora de la competencia social del alumnado de Educación Infantil y Primaria
}

\section{Guadalupe Alba Corredor, Ana Justicia-Arráez, M. Carmen Pichardo Martínez y Fernando Justicia Justicia}

Departamento Psicología Evolutiva y de la Educación.

Universidad de Granada

\section{España}




\section{Resumen}

Los problemas de conducta son una realidad constatada en las aulas. Se confirma, por una parte, que las edades en las que aparecen son cada vez más tempranas y, por otra, que no existen intervenciones que garanticen la disminución de este tipo de conductas, dirigidas a la población infantil. Tanto las investigaciones como las evidencias previas al respecto ponen de manifiesto la relevancia de trabajar la competencia social con el alumnado de Educación Infantil y Primaria como factor de protección frente a los problemas de conducta. Frente a este problema social y educativo, países norteamericanos y europeos han dado respuesta a través de la inclusión en el curriculum de programas de prevención universal que garantizan el aprendizaje de la competencia social en las escuelas.

En este sentido, el programa Aprender a Convivir, pionero en este ámbito, pretende ser una intervención innovadora y preventiva que favorece el óptimo desarrollo de conductas prosociales en niños y niñas de tres a siete años. A través de la inclusión de contenidos tales como normas, sentimientos y emociones, empatía, habilidades de comunicación, habilidades de interacción personal, asertividad o resolución de conflictos, entre otros, se favorece el aprendizaje de la competencia social a la vez que se reducen los problemas de conducta. Hecho que, a largo plazo, garantiza la prevención del comportamiento antisocial.

La presente aportación pretende, por un lado, poner de manifiesto la importancia de trabajar desde las escuelas la competencia social y, por otro, dar a conocer el programa Aprender a Convivir, una propuesta educativa implementada en diferentes ciudades españolas (Granada, Zaragoza) además de en otros países de habla hispana (Bolivia, República Dominicana), que ha obtenido resultados significativos para el aprendizaje de la competencia social y la reducción de problemas de conducta.

Palabras clave: programa prevención universal, competencia social, habilidades sociales, problemas de conducta, Educación Infantil y Educación Primaria. 


\title{
Aprender a Convivir. A prevention program for improving social competence in preschool and elementary school children
}

\begin{abstract}
Behavior problems are usually observed at classrooms. First of all, the age in which problems appear, is increasing in an early way, and on the other hand, there are no interventions that ensure the reduction of such conduct problems for school age children. Research and previous evidence show the relevance of social competence in this kind of prevention. Working with kindergarten and elementary students must be the main objective, because social competence is a protective factor against behavior problems. Due to these social and educational problems, Americans and Europeans countries have responded through the inclusion in the curriculum of universal prevention programs to guaranteethe learning of social competence at schools.
\end{abstract}

In this sense, the program Aprender a Convivir, pioneer in this field, can be considered as a preventive intervention that encourages the innovative and optimal development of prosocial behavior in three to seven year-old children. The main contents are: rules, feelings and emotions, empathy, communication skills, interpersonal skills, assertiveness or problem solving skills. The framework is focused on the increasing of social competenceand the decreasing of behavior problems; fact that, in a long term, produces the prevention of antisocial behavior.

The present contribution aims to show the importance of working social competence from schools, and secondly, to present the program Aprender a Convivir which has being carried out in different Spanish cities (Granada, Zaragoza), and in another Spanish-speaking countries (Bolivia, Dominican Republic) as well, showing significant differences in the growth of social competence and the reduction of behavior problems.

Key words: Universal prevention program, social competence, social skills, behavioral problems, conduct problems, preschool education, kindergarten and elementary school. 


\section{Introducción}

El programa Aprender a Convivir surge del interés común de varios investigadores por dar respuesta a una problemática social incipiente: la necesidad de educar en competencia social.

En las últimas décadas se han incrementado considerablemente las investigaciones sobre violencia escolar (Cava, Buelga, Musitu y Murgui, 2010). Este tipo de conducta encierra comportamientos transgresores que tienen lugar en los colegios e institutos. Abarcan desde actos delictivos leves, hasta patrones de comportamiento más graves relacionados con la agresión física y verbal a compañeros y profesores (Cava y Musitu, 2002; Díaz-Aguado, Martínez y Martín, 2004; Olweus, 1998). Todas estas conductas impiden el normal desarrollo de la enseñanza y afectan gravemente al clima escolar y a las relaciones interpersonales del profesorado y del alumnado (Del Prette y Del Prette, 2009; Del Prette, Del Prette, De Olivieira, Gresham yVance, 2012; Gresham, 2009; Trianes, 2000). No obstante, y pese a que las situaciones de violencia y maltrato entre compañeros son las que han generado una mayor atención por parte de los investigadores y una mayor preocupación por parte de la sociedad (Caballero y Huertas, 2013; Defensor del Pueblo, 2007; Letamendía, 2002), se deben revisar todos los comportamientos que inciden negativamente en la convivencia escolar.

Desde hace unas décadas, tal y como se recoge en diferentes informes, la situación educativa en materia de convivencia escolar ha degenerado. Las conductas agresivas y disruptivas se producen cada vez a edades más tempranas manteniéndose durante la infancia y adolescencia (Caspi, Moffitt, Newman y Silva, 1996; Hofstra, van der Ende y Verhulst, 2002; Loeber, Burke, Lahey, Winters y Zera, 2000; Moffitt, Caspi, Harrington y Milne, 2002; Tremblay, Pihl, Vitaro y Dobkins, 1994).

Tal y como afirma Aragón et al. (2003), la conducta agresiva cuando persiste más allá de tercero de Educación Primaria es, quizás, el diagnóstico que comporta mayor riesgo de futuros problemas en la adolescencia, prediciendo conducta antisocial, abandono del colegio, adicciones y conducta predelictiva. Es por ello importante tratar de reducir y prevenir este tipo de comportamiento desde edades tempranas. Numerosas investigaciones señalan la relación existente entre los problemas de conducta y la competencia social, considerándose esta últi- 
ma, factor de protección frente a la aparición de diversos comportamientos desadaptativos (Farrington, 2005; Ostrov et al., 2009; Patterson, Capaldi y Bank, 1991)

Existe un alto grado de consenso en la idea de que la competencia social, como factor de protección de las relaciones entre iguales en la infancia, contribuye significativamente al desarrollo del correcto funcionamiento interpersonal y proporciona oportunidades únicas para elaprendizaje de habilidades específicas que no pueden lograrse de otra manera ni en otros momentos (Amesty y Clinton, 2009; Monjas y González, 2000).

\section{La competencia social}

Tal y como afirma Vaughnet al. (2009) hay consenso entre los científicos a cerca del sentido que tiene la competencia social. Se entiende por competencia social la capacidad para utilizar ciertas habilidades necesarias cuando se interactúa con los demás, a saber, es la aptitud para las relaciones interpersonales (entiéndase la aptitud como capacidad para operar competentemente en una determinada actividad) que conlleva el uso de una serie de habilidades. Se trata de un atributo con una importancia crucial en la edad preescolar, ya que se asume que es la base del ajuste interpersonal del niño en la escuela (Coombs, Myran y Tonelson, 2009; Del Prette y Del Prette, 2010; Ladd, 2005). Sin embargo, y a pesar de este consenso, hay menos acuerdo dentro de la comunidad científica, sobre cómo debe establecerse la competencia social en la edad preescolar y lo que significa ser socialmente competente en las diversas etapas del desarrollo evolutivo del niño.

\section{El aprendizaje de la competencia social}

Parece que existe en los niños una tendencia hacia el comportamiento prosocial. Tomasello (2010) constata en sus estudios que niños y niñas, desde edades muy tempranas, muestran conductas sociales adaptadas que van más allá de sus propios intereses. Estas conductas se siguen manteniendo e incrementando dentro del sistema familiar gracias a diferentes modelos de aprendizaje, a través de los cuales el alumnado desarrolla la competencia social: a) aprendizaje por experiencia directa, b) aprendizaje por observación, c) aprendizaje verbal o instruccional y d) aprendizaje por feedback interpersonal (Monjas y González, 2000).

Esta predisposición junto con este aprendizaje favorable de aptitudes, ayuda a consolidar el comportamiento social, pero esta incipiente capacidad puede verse perjudicada por la existencia de factores individuales, familiares o contextuales que ponen en riesgo el desarrollo 
normal de la misma. Por lo tanto, es necesario que la competencia social sea enseñada de manera más explícita e intencional (Bredekamp y Coople, 1997). El período de Educación Infantil y los primeros años de Educación Primaria son el mejor momento para llevar a cabo una intervención óptima que facilite la adquisición de la competencia social (Webster-Stratton y Reid, 2004).

La evidencia previa como manifestación de la necesidad de programas de competencia social

A pesar de los notables progresos realizados en la última década en pro del desarrollo y la difusión de enfoques basados en la prevención dentro de la escuela (Detrich, Keyworth y States, 2008) todavía hoy, sigue siendo insuficiente el nivel de evidencia de la eficacia de coordinar intervenciones en la primera infancia que aborden los múltiples factores de riesgo y las condiciones que colocan al niño ante situaciones que más tarde tendrán resultados destructivos, tales como la delincuencia o el abuso de drogas. Hoagwood y colaboradores han argumentado que la adopción, la sostenibilidad y la integración de las intervenciones eficaces entre las prácticas normales de las escuelas y en los entornos clínicos aún no han sido demostradas con suficiente amplitud (Hoagwood, Kerker, Kratochwill, Crowe, y Saka, 2007).

Prevenir, reducir y detener el comportamiento agresivo en los primeros años de escolarización, cuando el comportamiento del alumnado es muy maleable, es más beneficioso y menos costoso que interrumpir la progresión que comienza con problemas de conducta a edades tempranas y termina con fracaso escolar y delincuencia en edades posteriores (WebsterStratton y Reid, 2004).

\section{Los programas de entrenamiento en competencia social}

Las estrategias de prevención universal que se han utilizado para la enseñanza de los aprendizajes anteriormente mencionados se han etiquetado como entrenamiento. Entrenar supone preparar para la práctica. Así, el aprendizaje de la competencia social conlleva la modificación de conductas que permiten un adecuado ajuste social. Sin embargo, esto no quiere decir que la competencia social quede reducida a un conjunto de técnicas o conductas moleculares (López de Dicastillo, Iriarte y González-Torres, 2006). Es necesario que se tengan en cuenta todos los procesos que intervienen en su adquisición, esto es, procesos cognitivos, sociales, emocionales y morales (Trianes y Muñoz, 2003). 
Diferentes investigaciones muestran que el entrenamiento de niños y niñas en competencia social es efectivo para la enseñanza de conductas socialmente hábiles (January, Casey y Paulson, 2011; Pickens, 2009; Pizato, 2010). Actualmente, se dispone de suficiente evidencia sobre técnicas, estrategias y procedimientos que se pueden utilizar para la enseñanza de conductas que favorecen la interacción social en la infancia (Barreto, Freitas y Del Prette, 2011; Conduct Problems Prevention ResearchGroup, 2010; Hundert, 1995; Washburn et al., 2011). El problema radica en que la mayoría de los procedimientos o no son lo suficientemente sistemáticos para asegurar un buen aprendizaje social, o los que lo son, no son suficientes para garantizar el conocimiento de su existencia. A continuación, en la tabla 1 se muestran algunos programas de prevención universal nacionales e internacionales, destinados a trabajar la competencia social en el alumnado de Educación Infantil (en adelante, EI) y Educación Primaria (en adelante, EP).

Tabla 1

Programas de entrenamiento en competencia social parauna prevención universal en el alumnado de Educación Infantil y Educación Primaria

\begin{tabular}{|c|c|c|c|}
\hline & EDUCACIÓN INFANTIL & $\begin{array}{c}\text { EDUCACIÓN INFANTIL Y } \\
\text { EDUCACIÓN PRIMARIA } \\
\end{array}$ & EDUCACIÓN PRIMARIA \\
\hline \multirow{2}{*}{ ESCOLAR } & $\begin{array}{l}\text { Social-emotional intervention } \\
\text { for at-risk-4-years-olds } \\
\text { (Denham y Burton 1996) }\end{array}$ & $\begin{array}{l}\text { Relacionarnos Bien (Segura y Arcas, } \\
\text { 2004) }\end{array}$ & $\begin{array}{l}\text { Social CompetencyProgram } \\
\text { (Krasnow, 1992) }\end{array}$ \\
\hline & Emotions course (Izard, 2001) & $\begin{array}{l}\text { Propuesta de Curriculum Emocional en } \\
\text { Educación Infantil (Bisquerra, } \\
\text { Soldevilla,Ribes, Filella y Aguyó, 2005) }\end{array}$ & $\begin{array}{l}\text { Program for Academic } \\
\text { Survival Skills (Greenwood, } \\
\text { Hops y Walker, 1977) }\end{array}$ \\
\hline \multirow{6}{*}{$\begin{array}{l}\text { ESCOLAR- } \\
\text { FAMILIAR }\end{array}$} & $\begin{array}{l}\text { ICanProblem Solve (Shure, } \\
\text { 1993) }\end{array}$ & $\begin{array}{l}\text { ProjetPrima!r (PetermannyNatzke, } \\
2008)\end{array}$ & $\begin{array}{l}\text { Programa de Desarrollo } \\
\text { Social y Afectivo (Trianes, } \\
1996 \text { ) }\end{array}$ \\
\hline & $\begin{array}{l}\text { Second Step for Preschoolers } \\
\text { and Kindergartens } \\
\text { (Comiteeforchildren, 2002) }\end{array}$ & $\begin{array}{l}\text { Early Impact Program (Lamar, Dadds y } \\
\text { Shochet, 2006) }\end{array}$ & \\
\hline & \multirow[t]{4}{*}{$\begin{array}{l}\text { PATHS Preschool: Promoting } \\
\text { Alternative Thinking } \\
\text { Strategies (Domitrovich, } \\
\text { Greenberg, Kuschey } \\
\text { Cortes,2004) }\end{array}$} & $\begin{array}{l}\text { PALS Social Skills Program: Playing } \\
\text { andLearning to Socialise (Cooper, } \\
\text { Goodfellow, Muhlheim, Paske y } \\
\text { Pearson,2003) }\end{array}$ & \\
\hline & & $\begin{array}{l}\text { Programa de Enseñanza de Habilidades } \\
\text { de Interacción Social (PEHIS) (Monjas, } \\
2002 \text { ) }\end{array}$ & \\
\hline & & $\begin{array}{l}\text { Aprender a Convivir (Justica, Benítez, } \\
\text { Fernández, Fernández y Pichardo, 2008) }\end{array}$ & \\
\hline & & $\begin{array}{l}\text { El Programa para la Prevención } \\
\text { Temprana de la Violencia (Duque, } \\
\text { Orduz, Sandoval, Caicedo y Klevens, } \\
2007 \text { ) }\end{array}$ & \\
\hline
\end{tabular}


Tal y como pone de manifiesto la revisión realizada para el conocimiento de los programas, no existen muchas intervenciones, dentro del ámbito nacional, que tengan como objetivo desarrollar la competencia social del alumnado en los primeros años de escolarización. La finalidad de este artículo es dar a conocer el programa Aprender a Convivir desarrollado para el alumnado de Educación Infantil y Educación Primaria. Para ello se definirán los objetivos, se presentarán los contenidos, así como la estructura y la metodología a seguir, para concluir con la evaluación. Todos estos aspectos se organizarán de manera individual integrando cada una de las etapas en los diferentes elementos curriculares.

\section{El programa Aprender a Convivir}

\section{Objetivo}

Aprender a Convivir es un programa de intervención preventiva de tipo universal que tiene como objetivo principal desarrollar en el niño de tres a siete años una competencia social suficiente con la que poder abordar y solucionar situaciones de conflicto desde edades tempranas (Justicia, Benítez, Fernández, Fernández, y Pichardo, 2008). Es por ello, que dicha intervención está diseñada para implementarse durante el segundo ciclo de EI y primer ciclo de EP.

\section{Características del material}

Los contenidos del programa Aprender a Convivir se fundamentan en cuatro aspectos básicos: el constructo competencia social, el desarrollo evolutivo del niño, el curriculum de EI y EP en cada caso, y la evidencia previa manifestada por la revisión de programas. En el caso de EI la intervención se divide en cuatro bloques de contenidos: 1) Las normas y su cumplimiento; 2) Sentimientos y emociones; 3) Habilidades de comunicación, y 4) Ayuda y cooperación (véase Tabla 2).Cada bloque consta de tres unidades que se trabajan a razón de una unidad por semana, estando la unidad compuesta por dos sesiones. La duración semanal del programa es de una hora (media hora, por sesión) y la implementación total abarca 12 semanas del curso. 
Tabla 2. Contenidos del programa "Aprender a Convivir" para Educación Infantil

\begin{tabular}{|c|c|c|c|}
\hline \multirow{4}{*}{$\begin{array}{l}\text { Las normas y su } \\
\text { cumplimiento }\end{array}$} & 3 años & 4 años & 5 años \\
\hline & $\begin{array}{l}\text { Conocemos y cumplimos } \\
\text { las normas referentes a la } \\
\text { organización }\end{array}$ & $\begin{array}{l}\text { Conocemos la importan- } \\
\text { cia de las normas y las } \\
\text { cumplimos }\end{array}$ & $\begin{array}{l}\text { Conocemos el valor y la } \\
\text { importancia de las normas y } \\
\text { las cumplimos }\end{array}$ \\
\hline & $\begin{array}{l}\text { Conocemos y cumplimos } \\
\text { las normas referentes a las } \\
\text { relaciones interpersonales }\end{array}$ & $\begin{array}{l}\text { Conocemos y cumplimos } \\
\text { las normas referentes a la } \\
\text { organización y a las rela- } \\
\text { ciones personales }\end{array}$ & $\begin{array}{l}\text { Participamos en actividades } \\
\text { de juego y de clase cum- } \\
\text { pliendo la norma de atender } \\
\text { a profesores e iguales }\end{array}$ \\
\hline & $\begin{array}{l}\text { Comprendemos lo que } \\
\text { sucede cuando no se cum- } \\
\text { plen las normas estableci- } \\
\text { das }\end{array}$ & $\begin{array}{l}\text { Participamos en activida- } \\
\text { des de juego y de la casa, } \\
\text { cumpliendo las normas }\end{array}$ & $\begin{array}{l}\text { Identificamos y soluciona- } \\
\text { mos problemas que surgen } \\
\text { por no cumplir las normas }\end{array}$ \\
\hline \multirow{4}{*}{$\begin{array}{l}\text { Sentimientos y emo- } \\
\text { ciones }\end{array}$} & $\begin{array}{l}\text { Reconocemos sentimientos } \\
\text { y emociones propias }\end{array}$ & $\begin{array}{l}\text { Reconocemos sentimien- } \\
\text { tos y emociones básicas }\end{array}$ & $\begin{array}{l}\text { Reconocemos emociones } \\
\text { primarias y secundarias }\end{array}$ \\
\hline & $\begin{array}{l}\text { Expresamos positivamente } \\
\text { las emociones. La alegría }\end{array}$ & $\begin{array}{l}\text { Controlamos las emocio- } \\
\text { nes }\end{array}$ & $\begin{array}{l}\text { Controlamos las emociones } \\
\text { y el comportamiento }\end{array}$ \\
\hline & $\begin{array}{l}\text { Expresamos positivamente } \\
\text { las emociones. El enfado }\end{array}$ & $\begin{array}{l}\text { Reconocemos sentimien- } \\
\text { tos y emociones en los } \\
\text { demás }\end{array}$ & $\begin{array}{l}\text { Identificamos los estados } \\
\text { emocionales de los demás }\end{array}$ \\
\hline & $\begin{array}{l}\text { Conocemos la importancia } \\
\text { de saber escuchar }\end{array}$ & $\begin{array}{l}\text { Conocemos la importan- } \\
\text { cia de saber preguntar }\end{array}$ & $\begin{array}{l}\text { Expresamos lo que nos mo- } \\
\text { lesta. Gracias, de nada y por } \\
\text { favor }\end{array}$ \\
\hline \multirow[t]{3}{*}{$\begin{array}{l}\text { Habilidades de co- } \\
\text { municación }\end{array}$} & Pedimos perdón & $\begin{array}{l}\text { Expresamos lo que nos } \\
\text { molesta }\end{array}$ & $\begin{array}{l}\text { Aprendemos a sugerir, no } \\
\text { damos órdenes }\end{array}$ \\
\hline & Damos las gracias & $\begin{array}{l}\text { Pedimos las cosas de } \\
\text { forma adecuada }\end{array}$ & $\begin{array}{l}\text { Aprendemos a resolver pro- } \\
\text { blemas que surgen en la } \\
\text { comunicación }\end{array}$ \\
\hline & $\begin{array}{l}\text { Comprendemos la impor- } \\
\text { tancia y beneficios de com- } \\
\text { partir }\end{array}$ & $\begin{array}{l}\text { Comprendemos la impor- } \\
\text { tancia de compartir y } \\
\text { ayudar }\end{array}$ & $\begin{array}{l}\text { Aprendemos a compartir y } \\
\text { ayudar }\end{array}$ \\
\hline \multirow[t]{2}{*}{$\begin{array}{l}\text { Ayuda y coopera- } \\
\text { ción }\end{array}$} & $\begin{array}{l}\text { Aprendemos a compartir } \\
\text { las cosas con los demás }\end{array}$ & $\begin{array}{l}\text { Aprendemos a ayudar a } \\
\text { los demás }\end{array}$ & $\begin{array}{l}\text { Comprendemos la importan- } \\
\text { cia de cooperar con los de- } \\
\text { más }\end{array}$ \\
\hline & $\begin{array}{l}\text { Comprendemos el concepto } \\
\text { de ayuda, su importancia y } \\
\text { beneficios }\end{array}$ & $\begin{array}{l}\text { Ya sabemos ayudar. La } \\
\text { importancia de cooperar }\end{array}$ & $\begin{array}{l}\text { Aprendemos a trabajar y } \\
\text { ayudar en grupo }\end{array}$ \\
\hline
\end{tabular}

Por otra parte, el programa del primer ciclo de EP tiene como base lo trabajado en la etapa anterior pero con modificaciones sustanciales que vienen justificadas por los factores mencionados anteriormente. Estos cambios son debidos, en primer lugar, a la introducción de nuevos contenidos y, en segundo lugar, a la modificación de la estructura; ya no hay bloques 
sino que se trabaja por unidades y temas. Cada unidad está compuesta por un tema. La diferencia entre unidad y tema reside en el nivel de concreción curricular, las primeras se establecen en el nivel de ciclo, esto supone que son comunes para ambos cursos mientras que los temas son específicos de cada curso (véase Tabla 3). El programa se compone de 14 unidades, 14 temas, que se trabajan a razón de un tema a la semana. Cada tema consta de cuatro sesiones, la duración de éste está programada para que se realice en una hora y 45 minutos, la implementación total abarca 14 semanas del curso.

Tabla 3. Contenidos del programa "Aprender a Convivir" para Educación Primaria

\begin{tabular}{|c|c|c|}
\hline Unidades & Temas $1^{\circ}$ Educación Primaria & Temas $2^{\circ}$ Educación Primaria \\
\hline Normas & $\begin{array}{l}\text { Creamos condiciones favorables para la } \\
\text { convivencia }\end{array}$ & $\begin{array}{l}\text { Somos disciplinados y obedientes en el } \\
\text { cumplimiento de las normas }\end{array}$ \\
\hline Valores & Las personas nos respetamos & $\begin{array}{l}\text { La responsabilidad y la sinceridad son dos } \\
\text { valores básicos de nuestra convivencia }\end{array}$ \\
\hline Atención & Nuestro cuerpo se comunica & Escuchamos activamente \\
\hline $\begin{array}{l}\text { Conciencia emo- } \\
\text { cional }\end{array}$ & $\begin{array}{l}\text { Reconocemos nuestras emociones y la de } \\
\text { los demás }\end{array}$ & Expresamos nuestras emociones \\
\hline Autocontrol & Nos paramos y pensamos & Me paro, pienso y actúo \\
\hline Respeto & Vivimos con más personas & $\begin{array}{l}\text { Aceptamos la diversidad y somos solidarios } \\
\text { con los compañeros }\end{array}$ \\
\hline Empatía & Estamos pendientes de los demás & Nos ponemos en el lugar de los demás \\
\hline Asertividad & Decimos no de forma adecuada & Defendemos nuestros derechos \\
\hline Esperar el momento & $\begin{array}{l}\text { Decimos las cosas en el momento adecua- } \\
\text { do y pedimos disculpas si no lo hacemos }\end{array}$ & Aceptamos las críticas y el no por respuesta \\
\hline $\begin{array}{l}\text { Relaciones inter- } \\
\text { personales }\end{array}$ & Ayudamos, compartimos y cooperamos & $\begin{array}{l}\text { Colaboramos para trabajar y jugar en equi- } \\
\text { po }\end{array}$ \\
\hline Hacer amigos & Nos unimos a un grupo & $\begin{array}{l}\text { Damos la bienvenida a compañeros en } \\
\text { nuestro grupo }\end{array}$ \\
\hline Autoconcepto & Nos conocemos y sabemos que podemos & Nos valoramos para que nos valoren \\
\hline $\begin{array}{l}\text { Resolución de con- } \\
\text { flictos I }\end{array}$ & Resolución de conflictos I & Resolución de conflictos I \\
\hline $\begin{array}{l}\text { Resolución de con- } \\
\text { flictos II }\end{array}$ & Resolución de conflictos II & Resolución de conflictos II \\
\hline
\end{tabular}


En cuanto a la estructura del programa, en el caso de EI, todas las unidades están compuestas por dos sesiones. Las actividades pertenecientes a cada sesión se pueden ver en la figura 1 .

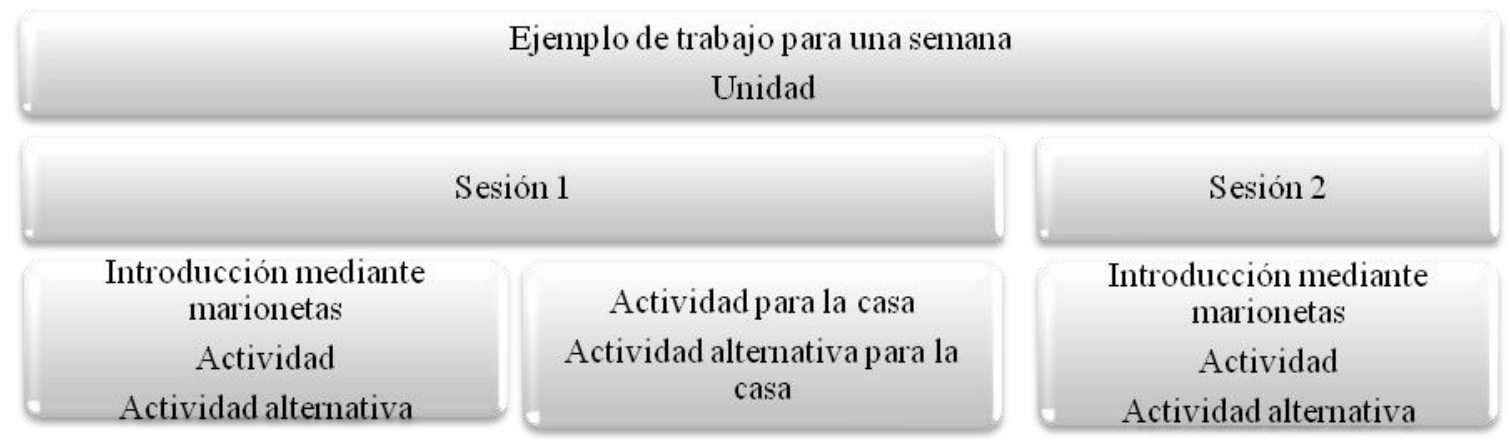

Figura 1. Estructura del programa en Educación Infantil

Por otra parte, cada uno de los temas de la intervención en EP se conforma por cuatro sesiones (véase Figura 2): 1) Introducción mediante viñetas, para presentar los objetivos que se van a trabajar en la sesión; 2) Jugando también se aprende, se trata de una sesión de Educación Física en la que se introducen y adaptan contenidos propios de ambos ámbitos; 3) Los cuentos nos enseñan algo, a través de la lectura crítica del mismo y 4) También hacemos en casa lo que hacemos en el colegio, el programa propone dos actividades para hacer con la familia con el objetivo de generalizar conductas a otros contextos.

\begin{tabular}{|c|c|c|c|}
\hline \multicolumn{4}{|c|}{$\begin{array}{c}\text { Ejemplo de trabajo para una semana } \\
\text { Tema }\end{array}$} \\
\hline Sesión 1 & Sesión 2 & Sesión 3 & Sesión 4 \\
\hline $\begin{array}{c}\text { Introducción mediante } \\
\text { viñetas } \\
\text { Actividad }\end{array}$ & $\begin{array}{c}\text { Jugando también se } \\
\text { aprende }\end{array}$ & $\begin{array}{l}\text { Los cuentos nos } \\
\text { enseñan algo }\end{array}$ & $\begin{array}{c}\text { También hacemos en } \\
\text { casa lo que } \\
\text { aprendemos en el } \\
\text { colegio }\end{array}$ \\
\hline
\end{tabular}

Figura 2. Estructura del programa en Educación Primaria.

\section{Metodología}

El sistema metodológico se entiende como el conjunto de estrategias de enseñanza y tareas de aprendizaje propuestas para la consecución de un objetivo. En este caso, el programa presenta una metodología global, activa e integrada que permite comunicar los contenidos 
fundamentales que merecen ser aprendidos de forma motivadora, respetando los principios didácticos de la EI y EP. Las estrategias de aprendizaje que se incluyen están representadas por una amplia variedad de técnicas o métodos que garantizan que todo el alumnado consiga el objetivo del programa.

En general, la base metodológica del programa, tanto en EI como en EP, está sustentada por la técnica de resolución de problemas. A saber, se fundamenta en elproceso a través del cual el niño descubre medios efectivos de enfrentarse asituaciones que se le plantean de manera cotidiana. Es un proceso estructurado que incluye identificación del problema, búsqueda de soluciones, análisis de las soluciones basado en las consecuencias de las mismas y toma de decisiones. Durante el segundo ciclo de EI este método es más flexible y se lleva a cabo de manera implícita. En cambio, en EP el alumnado trabaja la resolución de problemas, con la ayuda de un póster que explicita los distintos pasos del método, de forma más sistemática.

Por otra parte, también se utilizan otro tipo de estrategias como grupos cooperativos o role-playing, además de grupos de discusión y tormenta de ideas en EP. La forma en la que se organiza el grupo para la realización de las tareas de aprendizaje está en función de las mismas, pudiendo constituirse en gran grupo, pequeño grupo o trabajo individual. En el apartado siguiente se clarifican y analizan cada una de las tareas.

En cuanto a los recursos utilizados, decir que son muy variados. Entre ellos destacan las marionetas en EI y los cómics en EP. También se utilizan cuentos, juegos, canciones, murales, y otros materiales específicos para cada una de las actividades propuestas.

\section{Procedimiento de enseñanza}

En el período específico de implementación del programa, las técnicas y métodos de entrenamiento y enseñanza se articulan en una secuencia instruccional denominada procedimiento de enseñanza. Está compuesta por los siguientes elementos: instrucción verbal, diálogo y discusión; modelado; práctica; feedback; refuerzo y tareas (Goldstein, Sprafkin, Gershawy Klein, 1989; Monjas y Gónzález, 2000). Esta intervenciónsigue la secuencia instruccional anteriormente mencionada y denominada por diferentes autores como procedimiento de enseñanza, aunque el programa Aprender a Convivir añade a dicho procedimiento un apartado específico de evaluación. 
Instrucción verbal, diálogo y discusión

El primer apartado del procedimiento de enseñanza es la instrucción verbal, diálogo y discusión. Tiene como objetivo presentar y discutir la habilidad que se va a enseñar, a través de su presentación, diálogo en la resolución del problema y discusión sobre la relevancia que la habilidad tiene en los diversos contextos del alumnado. Se realiza a través de la actividad Introducción mediante marionetas (en EI) e Introducción mediante viñetas (en EP). En la tarea de EI una marioneta llamada Gavi (un gato) presenta el contenido de trabajo para la sesión. A continuación, Kike (niño) y Lupi (niña), otras dos marionetas, representan una situación problemática que deben resolver entrando en diálogo con el alumnado, para finalizar con la generalización de la habilidad a otros contextos como pueden ser la casa, el colegio o el patio. En cambio, en EP la presentación del contenido se realiza a través de un cómic donde los personajes presentan una situación y una solución que no tiene porqué ser adecuada, el alumnado debe buscar otras soluciones siguiendo la metodología de resolución de problemas.

\section{Modelado}

Tanto el profesorado como el alumnado representan, a través del role-playing, aspectos propios de las soluciones encontradas en la fase anterior. Generalmente, se lleva a cabo una ejecución previa con todo el grupo, donde el profesor muestra el ejemplo y los alumnos observan y repiten, posteriormente se realizanen pequeño grupo o por parejas. La fase del procedimiento no se realiza en todas las sesiones puesto que hay contenidos que tienen un carácter más cognitivo o afectivo, y son más difíciles de representar. Sin embargo, cuando esto ocurre, las marionetas o los personajes del cómic representan un modelo de actuación a imitar.

\section{Práctica}

Después de la presentación del contenido se realizan diferentes actividades de profundización de la habilidad presentada. Las tareas para el segundo ciclo de EI y el primer ciclo de EP son variadas incluyendo en su repertorio canciones, cuentos, fichas o juegos.

Tanto en EP como en EI existen una serie de actividades que se realizan en casa con el objetivo de generalizar los aprendizajes realizados en la escuela a otros contextos como el familiar. Con ello se pretende, además, involucrar a la familia en la enseñanza de la competencia social. 


\section{Feedback y reforzamiento}

Durante la adquisición de la competencia, el profesorado aporta información concreta sobre el proceso de aprendizaje y la consecución del objetivo de la tarea proporcionando reforzamiento.

\section{Tareas}

En esta fase el alumnado adquiere el compromiso de trabajar durante la semana de forma más específica la habilidad propuesta, con el fin de interiorizarla e incluirla dentro de su repertorio conductual. No obstante, el alumnado no debe olvidar aquellas habilidades aprendidas anteriormente, la función del profesorado es recordar contenidos anteriores y velar por el cumplimiento de los mismos.

\section{Evaluación}

Simultáneamente a todo el proceso de implementación del programa, se lleva a cabo un sistema de economía de fichas para evaluar los objetivos conseguidos en cada unidad o tema. El alumnado, guiado por el maestro, reflexiona sobre su comportamiento y la consecución del objetivo trabajado durante la semana.

Además de esta evaluación realizada por el alumno y guiada por el profesor, en el programa de EI, existe otra evaluación que realiza el profesorado en la que a través de un instrumento psicométrico que facilita el programa (Registro de Observación del programa Aprender a Convivir) se evalúa el nivel de adquisición de los contenidos del programa en cada una de las edades, mediante una escala de observación de 30 ítems con cuatro alternativas de frecuencia que oscilan entre $0=$ nunca hasta $3=$ siempre.

Frente a estos dos tipos de evaluación sumativa, realizadas a posteriori del aprendizaje, existe otro tipo de evaluación formativa en la que se comprueba la dinámica del proceso enseñanza/aprendizaje. En ella se analizan elementos relacionados con la organización, funcionamiento y aprovechamiento de las sesiones. 


\section{Conclusiones}

\section{Bondades y limitaciones}

Varias investigaciones recientes han puesto de relieve la necesidad de adoptar prácticas basadas en la evidencia que apoyen a los niños y niñas de edades tempranas en el aprendizaje de la competencia social.

En este trabajo se describe el programa de competencia social Aprender a Convivir, un programa que entrena un conjunto de habilidades sociales en el alumnado del segundo ciclo de EI y primer ciclo de EP. Este programa pretende dotar al niño de una serie de estrategias que le ayuden en sus relaciones sociales. Esta capacidad pasa porque el alumnado respete las normas y los límites que hacen posible una convivencia adecuada; tenga un conocimiento preciso de sus sentimientos y emociones, sepa reconocerlas en sí mismo y en los demás, así como que manifieste autocontrol y empatía. También se les enseña aspectos básicos en la comunicación con los otros, a ser asertivos, a defender sus derechos y a establecer relaciones positivas con los demás. Se les educa desde el conocimiento de su propia imagen y se les orienta a quererse y valorarse. Todo esto dentro de un contexto de aprendizaje en las relaciones interpersonales.

Como cualquier intervención, es más eficaz cuando se implementa con fidelidad y se mantienen la integridad de los contenidos, la secuencia y los métodos. Puesto que estos han sido diseñados teniendo en cuenta las características propias del niño de estas edades y temporalizados según los conocimientos previos necesarios. No obstante, como su finalidad pretende ser la inclusión en el curriculum, contiene la flexibilidad suficiente como para alterar, suprimir o ampliar los contenidos presentados.

El programa ha mostrado ser eficaz para la optimización de comportamientos prosociales y en la prevención de conductas disruptivas (Justicia et al., 2008; Fernández, 2010; Benítez, Fernández, Justicia, Fernández y Justicia-Arráez, 2011). Idealmente debe ser utilizado, de manera integrada, en un diálogo familia/escuela que permita el refuerzo común de las conductas y comportamientos que entrena. Aunque a las familias se les proporcionan recursos para este fin, estos pueden no ser suficientes. Una de las limitaciones de esta propuesta educativa es que no existe un programa de formación específico para los padres y madres, por lo que futuras líneas de investigación deberían ir encaminadas a la consecución de este objetivo. 
En definitiva, se trata de facilitar al profesorado recursos para la docencia que, de manera sistemática y con una evaluación previa, permitan abordar con garantía el proceso enseñanza/aprendizaje de la competencia social.

Propuestas de generalización y uso

Actualmente, el programa de EP se encuentra en fase de revisión y mejora. No así el programa de EI, que se halla en proceso de edición para ser presentado y difundido a los centros educativos. Tal y como se menciona, más arriba, en la introducción de este trabajo, el programa se orienta a un tipo de prevención universal del comportamiento antisocial en edades muy tempranas. No obstante, también se puede implementar en centros educativos pertenecientes a contextos de riesgo, así como hacia una intervención específica con alumnado con déficit de habilidades sociales.

Por último, también sería interesante poder aplicarlo en contextos no formales de aprendizaje como son los centros de recursos sociales, en asociaciones, en proyectos de atención a la infancia, en unidades de acción integral, en ludotecas, en acciones socioculturales, etc.

\section{Agradecimientos}

Este trabajo forma parte de un estudio más amplio integrado en varios Proyectos $\mathrm{I}+\mathrm{D}+\mathrm{i}$, Estudio longitudinal de los efectos del programa Aprender a Convivir en el desarrollo de la competencia social y la prevención del comportamiento antisocial (SEJ2005-04644) y Desarrollo de la competencia social en el alumnado de infantil y primaria como prevención del desarrollo de conductas de riesgo (EDU2009-11950), financiados por el Ministerio de Ciencia e Innovación y los Fondos Feder (Fondo Social Europeo). Asimismo, el estudio se ha realizado con el apoyo de una Beca de Formación de Profesorado Universitario (FPU), financiada por el Ministerio de Educación y Ciencia. 


\section{Referencias}

Amesty, E. y Clinton, A. (2009). Adaptación Cultural de un Programa de Prevención a Nivel Preescolar. Interamerican Journal of Psychology, 43(1), 106-113.

Aragón, E., García, B., Iglesias, A., Morena, L., Plata, E., Reche, C., Sánchez, A. y Trianes, M.V. (2003). Educar para la convivencia. En Proyectos de Investigación Educativa (pp. 105-110). Sevilla: Consejería de Educación y Ciencia. Junta de Andalucía.

Barreto, S. O., Freitas, L. C. y Del Prette, Z. A. P. (2011). Habilidades sociais na comorbidade entre dificuldade de aprendizagem e problemas de comportamento: Uma avaliação multimodal. PSICO, 43(1), 503-510.

Benítez, J. L., Fernández, M., Justicia, F., Fernández, E. y Justicia-Arráez, A. (2011). Results of the Aprender a Convivir. Program for the development of social competence and prevention of antisocial behavior in 4-year-old children. School Psychology International, 32(1), 3-19. doi:10.1177/0143034310396804

Bisquerra, R., Soldevilla, A. Ribes, R., Filella, G. y Aguyó, M. J. (2005). Una propuesta de curriculum emocional en Educación Infantil. Cultura y Educación, 17(1), 5-18. doi: $10.1174 / 113564007780191278$

Bredekamp, S., y Copple, C. (Eds.) (1997). Developmentally appropriate practice in early childhood programs. Washington, DC: National Association for the Education of Young Children.

Caballero Mariscal, D. y Huertas Delgado, F.J. (2013). Educación física y deporte como instrumento de resiliencia en menores inmigrantes. A tu salud, 81, 18-23.

Cava, M. J., Buelga, S., Musitu, G., y Murgui, S. (2010). Violencia escolar entre adolescentes y sus implicaciones en el ajuste psicosocial: un estudio longitudinal. Revista de Psicodidáctica, 15(1), 21-34.

Cava, M. J. y Musitu, G. (2002). La convivencia en la escuela. Barcelona: Paidós.

Caspi, A., Moffitt, T. E., Newman, D. L. y Silva, P. A. (1996). Behavioral observations at age of 3 predict adult psychiatric disorders: Longitudinal evidence from a birth cohort. $\begin{array}{llll}\text { Archives of } \quad \text { General } & \text { Psychiatry, }\end{array}$ doi:10.1001/archpsyc.1996.01830110071009.

Comitee for children (2002). Second Step: a violence prevention curriculum. Preschool/ Kindergarten. Seattle, WA: Comittee for Children.

Conduct Problems Prevention Research Group. (2010). The effects of a multi-year randomized clinical trial ofa universal social-emotional learning program: The role of student 
and school characteristics. Journal of Consulting and Clinical Psychology, 78(2), 156168. doi: 10.1037/a0018607

Coombs, R., Myran, S. P. y Tonelson, S. (2009). Teaching Social and Emotional Competence in Early Childhood. International Journal of Special Education, 24 (3), 143-149.

Cooper, J., Goodfellow, H., Muhlheim, E., Paske, K. y Pearson, L. (2003). PALS Social Skills Program: Playing and Learning to Socialise: Resource Book. Inscript Publishing.

Defensor del Pueblo (2007). Violencia escolar: El maltrato entre iguales en la Educación Secundaria Obligatoria 1999-2006. Madrid: Publicaciones de la Oficina del Defensor del Pueblo.

Del Prette, Z. A. P., y Del Prette, A. (2009). Psicologia das habilidades sociais na infância: Teoria e Prática. Rio de Janeiro: Vozes.

Del Prette, Z. A. P., y Del Prette, A. (2010). Habilidades Sociais e Análise do Comportamento: Proximidade histórica e atualidades. Perspectivas em Análise do Comportamento, $1(2), 104-115$.

Del Prette, Z. A. P., Del Prette, A., De Olivieira, L. A., Gresham, F. M. y Vance, M. J. (2012). Role of social performance in predicting learning problems: Prediction of risk using logistic regression analysis. School Psychology International Journal, 1(16), 615630.doi: 10.1177/0020715211430373

Denham, S. A. y Burton, R. (1996). A socio-emotional intervention for at risk 4-year-olds. Journal of School Psychology, 34(3), 225-245. doi: 10.1016/0022-4405(96)00013-1

Detrich, R., Keyworth, R., y States, J. (2008). Advances in evidence based education: A roadmap to evidence-based education. Oakland, CA: WingInstitute.

Díaz-Aguado, M. J., Martínez, R., y Martín, G. (2004). Prevención de la violencia y lucha contra la exclusión desde la adolescencia. Madrid: Ministerio de Trabajo y Asuntos Sociales, INJUVE.

Domitrovich, C., Greenberg, M., Kusche, C. y Cortes R. (2004). PATHS preschool program.South Deerfield, MA: Channing Bete Company.

Duque, L.F., Orduz, J.F., Sandoval, J.J., Caicedo, B.E., y Klevens, J. (2007). Lecciones del Programa de Prevención Temprana de la Violencia, Medellín, Colombia. Revista Panamericana de Salud Pública, 21(1), 21-29. doi: 10.1590/S102049892007000100003.

Farrington, D. (2005). Childhood origins of antisocial behavior.Clinical Psychology and Psychotherapy, 12, 177-190. Insertado 
Fernández, M. (2010). Desarrollo de la competencia social en niños de 3 años como prevención de problemas de conducta. (Tesis de doctorado, Universidad de Granada). Disponible en el repositorio DIGIBUG: Repositorio Institucional de la Universidad de Granada.

Greenwood, C. R., Hops, H., Walker, H. M. (1977). PASS: Program for Academic Survival Skills .Seattle, WA: Educational Achievement Systems.

Gresham, F. M. (2009). Análise do comportamento aplicada às habilidades sociais. En A. Del Prette, y Z. A. P. Del Prette (Eds.), Psicologia das habilidades sociais: Diversidade teórica e suasimplicações (pp. 17-66). Petrópolis: Vozes.

Goldstein, A. P., Sprafkin, R. P., Gershaw, N. J., y Klein, P. (1989). Habilidades sociales $\quad y$ autocontrol en la adolescencia. Barcelona: Martinez Roca.

Hoagwood, K. E., Olin, S. S., Kerker, B. D., Kratochwill, T. R., Crowe, M. y Saka, N. (2007). Empirically based school interventions targeted at academic and mental health functioning. Journal of Emotional and Behavioral Disorders, 15(2), 66-92. doi: $10.1177 / 10634266070150020301$

Hofstra, M. B., Van der Ende, J. y Verhulst, F. C. (2002). Pathways of self-reported problem behaviors from adolescence into adulthood. American Journal of Psychiatry, 159(3), 401- 407. doi:10.1176/appi.ajp.159.3.401

Hundert, J. (1995). Enhacing social competence in young students. Austin, TX: Pro-ed.

Izard, C. (2001). The emotion course: helping children understand and manage their feelings. Teachers manual. Newark D. E.: University of Deleware.

January, A. M., Casey, R. J. y Paulson, D. (2011). A Meta-analysis of Classroom-Wide intervention to Build Social Skills: Do They Work? Schoool Psychology Review, 40(2), 242-256.

Justicia, F., Benítez, J. L., Fernández, M., Fernández de Haro, E. y Pichardo, M. C. (2008). Aprender a convivir: programa de prevención do comportamento antisocial na educación infantil. Cadernos de psicoloxía, 32, 37- 47.

Krasnow, J. (1992). Parent-Teacher Action Research. Equity and Choice, 8(2), 35-39.

Ladd, G. W. (2005). Children's peer relations and social competence: A century of progress. New Haven, CT: Yale University Press.

Larmar, S., Dadds, M. y Shochet, I. (2006). Success and challenges in preventing conduct problems in Australia preschool-aged children through the Early Impact (EI) Program. Behaviour Change, 23(2), 121-137. doi: 0.1375/bech.23.2.121. 
Letamendía, R. (2002). El maltrato en contextos escolares. Revista de Psicodidáctica, 13, 7790.

Loeber, R., Burke, J.D., Lahey, B.B., Winters, A. y Zera, M. (2000). Oppositional defiant and conduct disorder: A review of the past 10 years, part I. Journal of the American Academy of Child and Adolescent Psychiatry, 39, 1468-1484. doi: 10.1097/0000458320001200000007

López de Dicastillo, N., Iriarte, C. y González-Torres, M.C. (2006). La competencia social y el desarrollo de comportamientos cívicos: la labor orientadora del profesor. Estudios sobre Educación, 11, 127-147.

Moffitt, T. E., Caspi, A., Harrington, H., y Milne, B.J. (2002). Males on the life-course persistent and adolescence-limited antisocial pathways: Follow-up at age 26. Development and Psychopathology, 14, 179-206.

Monjas. I. (2002) PEHIS: Programa de enseñanza de las habilidades de interacción social. Madrid: CEPE.

Monjas, I y González, B. (Dirs.) (2000). Las habilidades sociales en el currículo. Madrid: Secretaría General Técnica. Centro de Publicaciones. Ministerio de Educación, Cultura y Deporte.

Olweus, D. (1998). Conductas de acoso y amenaza entre escolares. Madrid: Morata.

Ostrov, J. M., Massetti, G. M., Stauffacher, K., Godleski, S. A., Hart, K. C., Karch, K. M. ... Ries, E. E. (2009). An intervention for relational and physical aggression in early childhood: A preliminary study. Early Childhood Research Quarterly, 24, 15-28. doi: 10.1016/j.ecresq.2008.08.002

Patterson, G. R., Capaldi, D. M. y Bank, L. (1991). An early started model for predicting delinquency. En D. J. Pepler y K.H. Rubin (Eds.), The development and treatment of Childhood aggression. Hillsdale, NY: Lawrence Erlbaum.

Petermann, F. y Natzke, H. (2008). Preliminary results of a comprehensive approach to prevent antisocial behavior in preschool and primary school pupils in Luxembourg. School Psychology International, 29(5), 606-626. doi: 10.1177/0143034308099204

Pickens, J. (2009). Socio-emotional Programme Promotes Positive Behaviour in Preschoolers.ChildCare in Practice, 15(4), 261-278. doi: 10.1080/13575270903149323

Pizato, E. C. G. (2010). Um estudo longitudinal de trajétorias de desempenho escolar. Tesis de doctorado. Universidad de Saõ Paulo.

Segura, M. y Arcas, M. (2004).Relacionarnos bien. Programa de competencia social para niños de 4-12 años. Madrid: Narcea. 
Shure, M. B. (1993). I can problem solve (ICPS): Interpersonal cognitive problem solving for young children. Early Child Development \& Care, 96, 49-64. doi: $10.1080 / 0300443930960106$

Tomasello, M. (2010). ¿Por qué cooperamos? Madrid: Katz Editores.

Tremblay, R. E., Pihl, R. O., Vitaro, F., y Dobkin, P. L. (1994). Predicting early onset of male antisocial behavior from preschool behavior. Archives of General Psychiatry, 51, 732-739. doi: 10.1001/archpsyc.1994.03950090064009

Trianes, M.V. (1996). Educación y competencia social. Un programa en el aula. Málaga: Aljibe.

Trianes, M. V. (2000). La violencia en contextos escolares. Málaga: Aljibe.

Trianes, M.V. y Muñoz, A. (2003). Educación de las habilidades sociales. En J. L. Gallego y E. Fernández de Haro (Dirs.) Enciclopedia de Educación Infantil, Vol. II (p. 197-218). Málaga: Aljibe.

Vaughn, B. E., Shin, N., Kim, M., Coppola, C., Krzysik, L, Santos, A. J., ...Korth, B. (2009). Hierarchical Models of Social Competence in Preschool Children: A Multisite, Multinational Study. Child Development, 80(6), 1775-1796. doi: 10.1111/j.14678624.2009.01367.x

Washburn, I. J., Acock, A., Vuchinich, S., Snyder, F., Li, K. K., Ji, P. ... Flay, B. R. (2011). Effects of a social-emotional and character development program on the trajectory of behaviors associated with social-emotional and character development: findings from three randomized trials. Prevention Science, 12(3), 314-23. doi: 10.1007/s11121-0110230-9.

Webster-Stratton, C., y Reid, M.J. (2004). Strengthening social and emotional competence in young children - the foundation for early school readiness and success: Incredible Years Classroom Social Skills and Problem-Solving Curriculum. Journal of Infants and Young Children, 17, 196-113. 
Guadalupe Alba Corredor et al.

[Página en blanco por razones de paginación]

- 904 - $\quad$ Electronic Journal of Research in Educational Psychology, 11(3), 883-904. ISSN: 1696-2095. 2013, no. 31 http://dx.doi.org/10.14204/ejrep.31.13105 